John Carroll University

Carroll Collected

2019 Faculty Bibliography

Faculty Bibliographies Community Homepage

2019

\title{
Descriptive Profiles of the MMPI-2-Restructured Form (MMPI-2-RF) across a National Sample of Four Veteran Affairs Treatment Settings
}

Paul Ingram

Texas Tech University

Anthony M. Tarescavage

John Carroll University, atarescavage@jcu.edu

Yossef S. Ben-Porath

Kent State University

Mary E. Oehlert

Eastern Kansas Veteran Healthcare System

Follow this and additional works at: https://collected.jcu.edu/fac_bib_2019

Part of the Psychology Commons

\section{Recommended Citation}

Ingram, Paul; Tarescavage, Anthony M.; Ben-Porath, Yossef S.; and Oehlert, Mary E., "Descriptive Profiles of the MMPI-2-Restructured Form (MMPI-2-RF) across a National Sample of Four Veteran Affairs Treatment Settings" (2019). 2019 Faculty Bibliography. 56.

https://collected.jcu.edu/fac_bib_2019/56

This Article is brought to you for free and open access by the Faculty Bibliographies Community Homepage at Carroll Collected. It has been accepted for inclusion in 2019 Faculty Bibliography by an authorized administrator of Carroll Collected. For more information, please contact connell@jcu.edu. 


\title{
Descriptive Profiles of the MMPI-2-Restructured Form (MMPI-2-RF $\begin{gathered}\text { Check for } \\ \text { updates }\end{gathered}$ across a National Sample of Four Veteran Affairs Treatment Settings
}

\begin{abstract}
This investigation provides descriptive information on substantive scale scores from the Minnesota Multiphasic Personality Inventory-2-Restructured Form (MMPI-2-RF) across four common service locations within Veterans Affairs (VA): PTSD clinical team, individual substance use treatment, primary medical care, and residential polytrauma rehabilitation. Test protocols for these four service settings are drawn from a national sample of all MMPI-2-RF and converted MMPI-2 administrations between January 1, 2008 and May 31, 2015 using the VA Mental Health Assist system at any VA across the United States. Frequency of substantive scale elevation and descriptive findings are examined. Results of this investigation suggest that there are differences between VA service locations on the MMPI-2-RF substantive scales, the magnitude of difference depends on the substantive scale examined, and the pattern of elevation within service location follows common clinical concerns for the settings. Implications for the clinical use, and research with, the MMPI-2-RF within the VA and with the veteran population are discussed.
\end{abstract}

Keywords MMPI-2-RF · Veteran affairs · Psychological assessment · Veteran

The Department of Veteran Affairs (VA) provides essential psychological and medical services to a sizable portion of the country's veterans. As of 2016, the VA offered these services at 1221 outpatient sites, 300 veteran centers, and 144 VA hospitals (United States Department of Veterans Affairs National Center for Veterans Analysis and Statistics 2017). Of the projected 21.68 million veterans in the country during 2015 , more than one in every three were enrolled in services at the VA. Moreover, roughly half of all veterans have utilized at least one service offered by the VA during any given year (National Center for Veterans Analysis and Statistics 2016). This high frequency of care and enrollment in services has steadily risen for the past 15 years, with roughly a quarter of all veterans now linked with the VA because of injuries associated with their service (Bagalman 2014). Much like enrollment, disability connection and associated care has also seen an increase in recent years (Duggan et al. 2010). In short, the VA is critical to promoting and improving veteran health on the national stage (e.g., Wilson and Kizer 1997).

The VA provides services for a distinctive population facing numerous barriers to good health and well-being. Some frequent obstacles to receiving needed care include a variety of resource shortages, such has having less optimal insurance coverage or a more limited ability to pay for needed services (Nelson et al. 2007; Wilson and Kizer 1997). In addition to these resourcebased difficulties, veterans also have substantially greater mental and physical health problems than the general public, which makes achieving and promoting health more difficult. Those using the VA are likely to need healthcare services more frequently (Agha et al. 2000) because of higher rates of substance use (Wagner et al. 2007), worse mental health (Hankin et al. 1999; Hoge et al. 2006; Kaplan et al. 2007; Seal et al. 2007), and more frequent and severe physical ailments (Kazis et al. 1998).

Increased veteran service utilization may be due, in part, to the VA's increased emphasis on evidenced-based treatment and symptom assessment (Shekelle et al. 2010), including the use of measurement-based care (Fortney et al. 2017). 
This emphasis on improved mental health screening and symptom identification is needed as there are several major health epidemics among veterans attracting major attention, including suicide (Kang et al. 2015) and post-traumatic stress disorder (PTSD) (Kilpatrick et al. 2013). With VA services utilized more (42-146\%) by those qualifying for a psychiatric diagnosis (Cohen et al. 2010), mental health assessments offer a means to screen for these and other mental health concerns and determine needed treatment (Seal et al. 2007, 2008).

Frequently, brief face-valid single symptom inventories (e.g., PTSD Checklist, Patient Health Questionnaire) are used to assess and track patient symptoms. Despite some ability to assist in diagnostic efforts and short administration times, there are also concerns about the effectiveness of such tools because of problematic false positive/false negative rates (Nelson et al. 2014). Broadband inventories, on the other hand, tend to evidence stronger psychometrics as they typically undergo more extensive validation processes that yield more precise and reliable symptom measurement. Broadband instruments also measure an array of mental health constructs rather than a single area of concern (e.g., PTSD, depression, etc.) which makes broadband inventories well suited to assess the numerous and often comorbid clinical concerns common to veterans (e.g., Seal et al. 2009). Finally, brief screening instruments do not have embedded protocol validity indicators, yet it is important to consider response style to ensure self-report information is not embellished, minimized, or provided by an individual who does not understand the test content. This need for the monitoring of response style is particularly pronounced within the VA (Ray 2017; Russo 2013). Between $33 \%$ to $53 \%$ of veterans undergo the forensically-intermingled compensation evaluation process (DeViva and Bloem 2003; Freeman et al. 2008) and those evaluations can incorporate historic testing data from the veteran's record (Worthen and Moering 2011) that may not reflect symptom presentation accurately if not screened for patterns of invalid responding. Thus, broadband measures offer a level of utility not available with brief symptom inventories and are well-suited to the task of aiding VA measurement-based care.

The Minnesota Multiphasic Personality InventoryRestructured Form (MMPI-2-RF; Tellegen and Ben-Porath 2008/2011) is a widely-used broadband instrument within the VA and research has consistently supported the reliability and validity of scores on MMPI-2-RF scales in veterans and military service members (e.g., Russo 2018). The validity scales consistently discriminate between valid and invalid patterns of responding (Goodwin et al. 2013; Nelson et al. 2011; Sellbom et al. 2012b; Shura et al. 2017), offering a means to evaluate the potential for response bias within the VA (Ray 2017). Similarly, the substantive scales are useful in predicting diagnostic presentations and identifying important clinical concerns (Koffel et al. 2012; Gottfried et al. 2014;
Sellbom et al. 2012a; Wolf et al. 2008). Moreover, the substantive scales have also been shown to predict treatment engagement (Anestis et al. 2015; Arbisi et al. 2013) and other treatment outcomes (Arbisi et al. 2008; Forbey et al. 2012).

Consistent with evidence of its clinical utility with veterans, the MMPI-2-RF Technical Manual includes two VA comparison groups (Tellegen and Ben-Porath 2008/2011). The first is from an inpatient substance use treatment unit and the second is from an inpatient psychiatric ward, providing important information about typical presentation within those settings. However, these comparison groups were derived from MMPI-2 test administrations in the 1990's and the interpretive context for recent assessments differs substantially, particularly given the rise of compensation processes (Bagalman 2014; Ray 2017). Likewise, the demographic characteristics of those samples under-represent or exclude several groups frequently cared for within the VA. For example, the substance use comparison group is comprised of only males and era of service underrepresents Gulf War veterans. Most importantly, the comparison groups reported in the Technical Manual represent a narrow band of VA clinical contexts and variability in response style across service locations is common (Ingram et al. 2019).

In light of the various distinct evaluative settings common within the VA and the emphasis placed on empirically-based assessment as part of measurement informed care, an examination of MMPI-2-RF scores across common VA treatment settings is needed. To accomplish this goal, this study reports substantive scale scores across four common VA treatment settings. Specifically, we report the descriptive characteristics of scale scores, the frequencies of veterans who produced clinically elevated scores on MMPI-2-RF substantive scales, and effect sizes that describe the magnitude of differences between settings for each substantive scale.

\section{Method}

\section{Participants}

All cases where a MMPI-2 or MMPI-2-RF were administered between January 1, 2008 and May 31, 2015 at any VA across the country were eligible for inclusion. Cases were included within this investigation only if their testing that was entered electronically into the VA Mental Health Assistant Suite and was accessible via the Corporate Data Warehouse (CDW) framework. A subset of four common VA treatment settings were identified for this study: individual therapy for substance use (SUD; $n_{\text {total }}=652, n_{\text {valid }}=260,60.1 \%$ invalid), PTSD 
clinical team (PCT; $n_{\text {total }}=4466, n_{\text {valid }}=2287,48.8 \%$ invalid), residential polytrauma and traumatic brain injury (Polytrauma; $n_{\text {total }}=1596, n_{\text {valid }}=612 ; 61.7 \%$ invalid $)$, and primary care medical settings (Medical; $n_{\text {total }}=$ $8711, n_{\text {valid }}=2247,74.2 \%$ invalid). Profiles were excluded if they were deemed uninterpretable, based on standard validity scale cut-scores (Ben-Porath and Tellegen 2008): CNS $\geq 18$, VRIN-r $\geq 80$, TRIN $\geq 80, F-r \geq 120$, and Fp-r $\geq$ 100 .

In general, those excluded from analysis most frequently demonstrate patterns of profile invalidity evidenced by elevations on the over-reporting scores as is consistently observed within the VA (see Ingram et al. 2019). Generally, valid participants across the four settings were demographically similar (Table 1). Veterans in this study tended to be service connected, male, married, and have served during Gulf War conflicts. Protocols drawn from PCT and Polytrauma were slightly more likely to be combat veterans and have been in a Gulf War conflict. These trends are most pronounced for Polytrauma. Those in SUD had higher rates of divorce and lower rates of marriage than other settings; they also had the greatest amount of missing data about service connection.

\section{Measures}

MMPI-2-Restructured Form The MMPI-2-RF (Ben-Porath and Tellegen 2008) is a 338 true-false item personality measure comprised of 51 scales. The 42 substantive scales measure various clinical constructs and the nine validity scales are used to determine if a respondent is engaging in non-credible responding (non-content based invalid responding, over-reporting, under-reporting). Although demonstrating large effect sizes, veteran status and issues critical to veterans (e.g., PTSD, traumatic brain injury, etc.) impact response style on the overreporting scales (Ingram and Ternes 2016). Given the forensically-enmeshed nature of the evaluation setting (Ray 2017), Ingram and colleagues (2019) recommend the use of the most conservative cut-scores identified within the interpretive manual for identifying invalid profiles for work within the VA: VRIN-r $\geq 80$, TRIN$r \geq 80$, F- $r \geq 120$, Fp- $r \geq 100$, Fs $\geq 100$, RBS $\geq 100$, FBS$r \geq 100$, L-r $\geq 80$, and K-r $\geq 70$. MMPI-2-RF scales scores calculated from the 567-item MMPI-2 test booklet are comparable to those calculated from the 338-item MMPI-2-RF booklet (c.f., Tarescavage et al. 2015).

\section{Procedures and Planned Analysis}

The CDW electronically stores veteran medical records from the entire United States. These data were extracted for this study through VA Informatics and Computing Infrastructure (VINCI). A request to the CDW was made based on testing administrations that were coded as including either a MMPI-2 or MMPI-2-RF. This study extracted demographic information as well as item-level responses for all identified administrations. Raw item responses were used to calculate MMPI-2RF scale scores. To identify different treatment modalities within the VA, we utilized stop codes (i.e., numerical values representing different clinics in which a service is conducted at the VA). Three of the four participant groups utilized a single stop code (513 SUD, 540 PCT, and 197 Polytrauma); however, to ensure a primary care medical group that represented both men and women, the women's primary care clinic (stop code 323 ) and internal medicine (stop code 301) were combined into a single Medical group. Information about the referral question(s) that led to evaluation was not available within the CDW.

Means and standard deviations were calculated for the scales of the MMPI-2-RF, as were frequencies with which the substantive scales exceeded T-scores recommended by the interpretative guidelines. Frequency of elevation was not calculated for the Validity Scales because all invalid protocols were excluded from analysis. For those comparison groups with enough women to form separate comparison groups $(n>=200)$, genderspecific comparison groups were calculated. Scale differences between the settings were also examined using Cohen's $d$ effect size estimates. Cohen (1988) proposed the following effect size classifications as guidelines for interpreting magnitude of effect: small (|.2| or less), medium (between $|.2|$ and $|.5|$ ), and large $(|.8|$ or greater).

\section{Results}

Descriptive statistics for the MMPI-2-RF substantive scales, as well as the frequency with which scale scores exceed recommended values $(T \geq 65)$, are provided in Tables 2 (Validity, Higher-Order, and Restructured Clinical) and 3 (Somatic, Cognitive, Internalizing, Externalizing, Interpersonal, and PSY-5). Also included in Tables 2 and 3 are the VA comparison group means described in the MMPI-2-RF Technical Manual (Tellegen and Ben-Porath 2008/2011). The samples from this current study do not have a direction indicated (T or F) on VRIN-r and TRIN-r because the descriptives were run on reflected T-scores. Across settings, veterans frequently exceeded clinical cut-scores for multiple scales. The scales that were the most pronounced within each group by scale mean and frequency of clinical elevation generally aligned with the expected clinical 
Table 1 Demographic characteristics of participants

\begin{tabular}{|c|c|c|c|c|}
\hline & $\begin{array}{l}\text { SUD } \\
n=260\end{array}$ & $\begin{array}{l}\text { PCT } \\
n=2287\end{array}$ & $\begin{array}{l}\text { Polytrauma } \\
n=612\end{array}$ & $\begin{array}{l}\text { Medical } \\
n=2247\end{array}$ \\
\hline \multicolumn{5}{|l|}{ Marital Status } \\
\hline Single / Separated & $81(31.2 \%)$ & $615(26.9 \%)$ & $159(26.0 \%)$ & $516(22.9 \%)$ \\
\hline Married & $65(25.0 \%)$ & $937(41.0 \%)$ & $308(50.3 \%)$ & $1088(48.4 \%)$ \\
\hline Divorced & $110(42.3 \%)$ & $689(30.1 \%)$ & $135(22.1 \%)$ & $534(23.8 \%)$ \\
\hline Widow/Widower & $3(1.2 \%)$ & $32(1.4 \%)$ & $2(0.3 \%)$ & $51(2.3 \%)$ \\
\hline Other/Missing & $1(0.4 \%)$ & $14(0.6 \%)$ & $8(1.4 \%)$ & $58(2.6 \%)$ \\
\hline \multicolumn{5}{|l|}{ Period of Service } \\
\hline World War II & - & $1(<0.0 \%)$ & - & $4(0.2 \%)$ \\
\hline Korea & - & $9(0.4 \%)$ & - & $12(0.5 \%)$ \\
\hline Vietnam-Era & $55(21.2 \%)$ & $673(29.4 \%)$ & $57(9.3 \%)$ & $835(37.2 \%)$ \\
\hline Post-Vietnam & $78(30.0 \%)$ & $301(13.2 \%)$ & $59(9.6 \%)$ & $196(8.7 \%)$ \\
\hline Gulf War & $123(47.3 \%)$ & $1287(56.3 \%)$ & $486(79.4 \%)$ & $1152(51.3 \%)$ \\
\hline Other/Missing & $4(1.6 \%)$ & $16(1.1 \%)$ & $10(1.6 \%)$ & $42(1.9 \%)$ \\
\hline \multicolumn{5}{|l|}{ Service Connection } \\
\hline$N S C$ & - & $322(14.1 \%)$ & $63(10.3 \%)$ & $267(11.9 \%)$ \\
\hline $0 \%$ & $5(1.9 \%)$ & $31(1.4 \%)$ & $5(0.8 \%)$ & $31(1.4 \%)$ \\
\hline $10-30 \%$ & $39(15.0 \%)$ & $208(9.1 \%$ & $63(10.4 \%)$ & $301(13.4 \%)$ \\
\hline $31-50 \%$ & $19(7.3 \%)$ & $212(9.3 \%)$ & $48(7.8 \%)$ & $307(13.7 \%)$ \\
\hline $51-70 \%$ & $37(14.3 \%)$ & $526(23.0 \%)$ & $106(17.3 \%)$ & $492(21.9 \%)$ \\
\hline $71-99 \%$ & $37(14.3 \%)$ & $537(23.5 \%)$ & $191(31.2 \%)$ & $528(23.5 \%)$ \\
\hline $100 \%$ & $31(11.9 \%)$ & $451(19.7 \%)$ & $136(22.2 \%)$ & $321(14.3 \%)$ \\
\hline Missing & $92(35.4 \%)$ & $322(14.1 \%)$ & - & - \\
\hline Male & $239(91.9 \%)$ & $1923(84.1 \%)$ & $528(86.3 \%)$ & $1968(87.6 \%)$ \\
\hline Combat Veteran & $26(10.0 \%)$ & $305(13.3 \%)$ & $120(19.6 \%)$ & $224(10.0 \%)$ \\
\hline
\end{tabular}

NSC Non service connected. This indicates that an individual was either not evaluated for service connection or that their evaluation indicated the presenting problem was not related to their military service. Information of education level is not coded within the electronic medical record and, thus, was not avaliable as demographic information. Age was not coded within the database used for analysis. SUD = individual substance use treatment, $\mathrm{PCT}=$ PTSD clinical teams, Polytrauma $=$ residential polytrauma units, Medical $=$ medical care settings. A indicates no records exist which match that cell's description concerns for that service location: SUD had the most frequent and pronounced elevations on scales associated with externalizing conduct problems and substance use (SUD, JCP, and BXD); Medical had notable somatic (MLS) and cognitively-focused (COG and NUC) elevations; Polytrauma mean elevations occurred on scales emphasizing a mixture of somatic (RC1, MLS) and neurological (NUC, COG) complaints, as well as a tendency towards social isolation (SAV, DSF); and PCT included internalizing (EID, RCd, RC2, RC7), avoidant (RC6, SAV, DSF), confused and disoriented thinking (THD, RC8), and trauma-like (AXY) elevations consistent with a presentation of posttraumatic stress disorder. PCT also had overall elevated means and clinical elevation frequencies across most scales, relative to the other groups Table 4.

Cohen $d$ effect sizes describing between-group differences are reported in Tables 5 (Validity, Higher-
Order, and Restructured Clinical scales) and 5 (Somatic/Cognitive, Internalizing, Externalizing, Interpersonal, and PSY-5 scales). Moderate to large effect sizes were frequent in comparisons between settings. Relative to the other settings, SUD had the most pronounced differences (represented by Cohen's $d$ values) on $\mathrm{BXD}$ (.43 to .61), $\mathrm{RC} 1$ ( -.46 to -.54$)$, RC4 (.50 to .78), SUD (.62 to 1.08 ), JCP (.38 to .48 ), and DISC-r (.48 to .67). Veterans on PCT were the most dissimilar from other service locations on $\mathrm{RCd}$ (.24 to .50), RC7 (.31 to .54), and AXY (.42 to .55). Polytrauma was distinct from other groups on EID (.21 to .56), HPC (.25 to .67), and DSF (.24 to .56). Those within the Medical group also had moderate to large effect differences from other groups; however, except for INTR-r (.62 to 1.03), effect sizes did not consistently indicate dissimilarity from all other groups. 
Table 2 Validity, Higher Order (H-O), and Restructured Clinical (RC) scale descriptive information

\begin{tabular}{|c|c|c|c|c|c|c|c|c|c|c|c|c|c|c|c|c|}
\hline \multirow[t]{3}{*}{ Scale } & \multicolumn{12}{|c|}{ Study Samples } & \multicolumn{4}{|c|}{ MMPI-2-RF Comparison Groups (VA Hospital, Men) } \\
\hline & \multicolumn{3}{|c|}{ SUD } & \multicolumn{3}{|c|}{ PCT } & \multicolumn{3}{|c|}{ Polytrauma } & \multicolumn{3}{|c|}{ Medical } & \multicolumn{2}{|c|}{ Psychiatric Inpatient } & \multicolumn{2}{|c|}{ Substance Abuse Treatment } \\
\hline & $M$ & $S D$ & $\% \geq 65$ & $M$ & $S D$ & $\% \geq 65$ & $M$ & $S D$ & $\% \geq 65$ & $M$ & $S D$ & $\% \geq 65$ & $M$ & $S D$ & $M$ & $S D$ \\
\hline Validity & - & - & - & - & - & - & - & - & - & - & - & - & - & - & - & - \\
\hline$V R I N-r$ & 54 & 10 & - & 52 & 10 & - & 52 & 10 & - & 52 & 10 & - & 53 & 10 & 54 & 10 \\
\hline$T R I N-r$ & 56 & 7 & - & 57 & 7 & - & 57 & 7 & - & 57 & 7 & - & $53 \mathrm{~T}$ & 1 & $52 \mathrm{~T}$ & 1 \\
\hline$F-r$ & 79 & 20 & - & 84 & 20 & - & 77 & 20 & - & 78 & 20 & - & 84 & 28 & 77 & 27 \\
\hline$F p-r$ & 62 & 14 & - & 66 & 14 & - & 61 & 14 & - & 62 & 14 & - & 62 & 15 & 59 & 15 \\
\hline Fs & 68 & 20 & - & 77 & 20 & - & 72 & 19 & - & 72 & 20 & - & 70 & 21 & 66 & 20 \\
\hline$F B S-r$ & 65 & 14 & - & 74 & 14 & - & 72 & 14 & - & 71 & 15 & - & 68 & 15 & 58 & 15 \\
\hline$R B S$ & 70 & 17 & - & 60 & 17 & - & 78 & 17 & - & 76 & 17 & - & 72 & 20 & 64 & 18 \\
\hline$L-r$ & 52 & 11 & - & 55 & 10 & - & 56 & 10 & - & 55 & 10 & - & 53 & 11 & 53 & 10 \\
\hline$K-r$ & 42 & 9 & - & 39 & 9 & - & 43 & 10 & - & 42 & 10 & - & 42 & 10 & 42 & 10 \\
\hline $\mathrm{H}-\mathrm{O}$ & - & - & - & - & - & - & - & - & - & - & - & - & - & - & - & - \\
\hline$E I D$ & 66 & 13 & 68.6 & 69 & 12 & 68.6 & 62 & 13 & 48.0 & 66 & 13 & 55.7 & 68 & 14 & 62 & 14 \\
\hline$T H D$ & 61 & 13 & 36.1 & 62 & 13 & 36.1 & 57 & 12 & 20.6 & 58 & 12 & 25.1 & 61 & 17 & 60 & 16 \\
\hline$B X D$ & 62 & 12 & 26.0 & 57 & 11 & 36.0 & 55 & 11 & 21.2 & 55 & 11 & 20.1 & 58 & 13 & 66 & 11 \\
\hline $\mathrm{RC}$ & - & - & - & - & - & - & - & - & - & - & - & - & - & - & - & - \\
\hline$R C d$ & 66 & 13 & 66.5 & 69 & 12 & 66.5 & 63 & 12 & 45.6 & 65 & 12 & 53.2 & 68 & 13 & 63 & 14 \\
\hline$R C 1$ & 65 & 13 & 72.1 & 72 & 13 & 72.1 & 72 & 13 & 72.4 & 71 & 13 & 68.6 & 65 & 15 & 62 & 15 \\
\hline$R C 2$ & 65 & 15 & 68.0 & 70 & 15 & 68.0 & 63 & 15 & 46.7 & 68 & 16 & 59.9 & 67 & 16 & 58 & 15 \\
\hline$R C 3$ & 57 & 12 & 35.3 & 58 & 12 & 35.3 & 56 & 11 & 28.3 & 57 & 12 & 31.5 & 55 & 12 & 60 & 12 \\
\hline$R C 4$ & 65 & 12 & 33.9 & 59 & 12 & 33.9 & 56 & 11 & 22.2 & 56 & 11 & 23.4 & 60 & 13 & 69 & 11 \\
\hline RC6 & 63 & 14 & 42.9 & 62 & 13 & 42.9 & 58 & 12 & 28.6 & 60 & 13 & 35.5 & 64 & 18 & 64 & 15 \\
\hline$R C 7$ & 61 & 13 & 55.9 & 65 & 13 & 55.9 & 58 & 13 & 34.3 & 61 & 13 & 43.5 & 61 & 14 & 59 & 14 \\
\hline$R C 8$ & 62 & 13 & 46.7 & 64 & 13 & 46.7 & 60 & 12 & 33.3 & 60 & 12 & 33.9 & 61 & 15 & 61 & 15 \\
\hline RC9 & 53 & 10 & 13.1 & 53 & 10 & 11.4 & 53 & 11 & 16.2 & 52 & 10 & 11.4 & 52 & 12 & 57 & 12 \\
\hline
\end{tabular}

Comparison group descriptives are reproduced from MMPI-2-RF Technical by Yossef S. Ben-Porath and Auke Tellegen. Copyright $\odot 2008,2011$ by the Regents of the University of Minnesota. Reproduced by permission of the University of Minnesota Press. All rights reserved. "Minnesota Multiphasic Personality Inventory-2-RF®" and "MMPI-2-RF®" are trademarks owned by the Regents of the University of Minnesota

Only the PCT and Medical groups had a sufficient sample size for calculating gender-specific comparison groups (Tables 6 and 7). Scores were generally similar for males and females on PCT with most effect sizes falling in the small to insignificant classification with most scales not exceeding thresholds for clinical significance. Of the minority of scales with medium to large effects $(n=6$; $11.5 \%$ ), females experienced greater internalizing symptoms (particularly related to fearfulness and self-image) and somatic concerns while males frequently had more externalizing scale elevations. The most notable differences existed for the DISC-r and AGGR-r scales across both mean and elevation frequency. The Medical group had a similar pattern with externalizing behaviors being higher in males and internalizing symptoms being lower in females; however, substantive differences of effect size were evident across a greater number of scales. Most differences were small although there were also more medium to large effects than seen in PCT.

\section{Discussion}

This study provides descriptive statistics for four common VA treatment settings using a national sample. Such an investigation was warranted as existing veteran comparison groups have limited generalizability. Existing comparison groups were established during the 1990's when the forensically enmeshed compensation procedures were less frequent. Prior comparison groups also under-represent frequently cared for groups within the VA and describe only a narrow band of available VA clinical settings. Accordingly, this study expands available comparison groups for veterans with the MMPI-2-RF and offers the most up-to-date representation of substantive scale responses currently observed within these settings. Findings from this descriptive analysis of the MMPI-2-RF substantive scales are discussed below and addressing four trends: (a) the substantive scale scores differ across VA service locations, 
Table 3 Somatic/cognitive, internalizing, externalizing, interpersonal, and PSY-5 scale descriptive information

\begin{tabular}{|c|c|c|c|c|c|c|c|c|c|c|c|c|c|c|c|c|}
\hline \multirow[b]{3}{*}{ Scale } & \multicolumn{12}{|c|}{ Study Samples } & \multicolumn{4}{|c|}{ MMPI-2-RF Comparison Groups (VA Hospital, Men) } \\
\hline & \multicolumn{3}{|c|}{ SUD } & \multicolumn{3}{|c|}{ PCT } & \multicolumn{3}{|c|}{ Polytrauma } & \multicolumn{3}{|c|}{ Medical } & \multicolumn{2}{|c|}{ Psychiatric Inpatient } & \multicolumn{2}{|c|}{ Substance Abuse Treatment } \\
\hline & $M$ & $S D$ & $\% \geq 65$ & $M$ & $S D$ & $\% \geq 65$ & $M$ & $S D$ & $\% \geq 65$ & $M$ & $S D$ & $\% \geq 65$ & $M$ & $S D$ & $M$ & $S D$ \\
\hline Somatic/Cognitive & - & - & - & - & - & - & - & - & - & - & - & - & - & - & - & - \\
\hline$M L S$ & 68 & 13 & 57.3 & 74 & 11 & 77.1 & 70 & 13 & 64.7 & 72 & 12 & 70.9 & 69 & 13 & 64 & 13 \\
\hline GIC & 59 & 15 & 28.8 & 67 & 16 & 48.5 & 62 & 16 & 36.1 & 65 & 17 & 41.5 & 61 & 17 & 58 & 16 \\
\hline$H P C$ & 60 & 12 & 38.8 & 65 & 12 & 56.4 & 68 & 12 & 64.7 & 64 & 12 & 52.7 & 59 & 13 & 57 & 13 \\
\hline$N U C$ & 65 & 14 & 55.4 & 70 & 14 & 70.7 & 72 & 14 & 75.3 & 70 & 14 & 71.0 & 66 & 16 & 62 & 16 \\
\hline$C O G$ & 68 & 15 & 55.0 & 72 & 14 & 68.2 & 75 & 13 & 77.6 & 69 & 15 & 58.6 & 67 & 16 & 61 & 16 \\
\hline Internalizing & - & - & - & - & - & - & - & - & - & - & - & - & - & - & - & - \\
\hline$S U I$ & 59 & 17 & 45.0 & 59 & 17 & 45.5 & 53 & 15 & 26.3 & 57 & 16 & 39.6 & 73 & 25 & 64 & 22 \\
\hline$H L P$ & 58 & 15 & 31.5 & 61 & 14 & 38.4 & 56 & 14 & 23.4 & 61 & 15 & 38.8 & 63 & 15 & 54 & 14 \\
\hline$S F D$ & 61 & 13 & 49.6 & 61 & 12 & 52.9 & 57 & 12 & 36.9 & 59 & 12 & 42.9 & 63 & 12 & 58 & 13 \\
\hline$N F C$ & 59 & 12 & 32.7 & 59 & 12 & 30.7 & 56 & 11 & 18.6 & 57 & 12 & 25.5 & 60 & 13 & 57 & 12 \\
\hline$S T W$ & 59 & 12 & 45.4 & 62 & 12 & 51.9 & 58 & 12 & 40.0 & 59 & 12 & 43.8 & 61 & 12 & 58 & 12 \\
\hline$A X Y$ & 70 & 17 & 61.9 & 78 & 16 & 80.7 & 69 & 17 & 59.3 & 71 & 17 & 63.2 & 67 & 19 & 62 & 17 \\
\hline$A N P$ & 58 & 12 & 33.8 & 63 & 12 & 56.7 & 60 & 13 & 45.1 & 61 & 13 & 45.7 & 58 & 13 & 56 & 13 \\
\hline$B R F$ & 57 & 13 & 23.8 & 59 & 13 & 25.6 & 55 & 12 & 30.2 & 57 & 13 & 17.9 & 57 & 13 & 56 & 12 \\
\hline$M S F$ & 48 & 9 & 8.5 & 49 & 9 & 9.2 & 46 & 7 & 2.9 & 48 & 9 & 8.0 & 49 & 9 & 51 & 9 \\
\hline Externalizing & - & - & - & - & - & - & - & - & - & - & - & - & - & - & - & - \\
\hline$J C P$ & 60 & 13 & 33.5 & 55 & 12 & 19.1 & 55 & 13 & 20.6 & 54 & 12 & 16.7 & 59 & 13 & 64 & 12 \\
\hline$S U B$ & 65 & 13 & 51.5 & 57 & 13 & 24.1 & 52 & 11 & 10.9 & 54 & 12 & 15.9 & 56 & 14 & 73 & 13 \\
\hline$A G G$ & 58 & 13 & 31.5 & 60 & 12 & 35.3 & 56 & 12 & 26.5 & 57 & 13 & 29.8 & 56 & 14 & 59 & 14 \\
\hline$A C T$ & 53 & 13 & 23.1 & 52 & 12 & 17.2 & 51 & 12 & 16.7 & 50 & 11 & 13.0 & 52 & 13 & 53 & 12 \\
\hline Interpersonal & - & - & - & - & - & - & - & - & - & - & - & - & - & - & - & - \\
\hline$F M L$ & 57 & 12 & 23.5 & 57 & 12 & 23.7 & 54 & 11 & 16.2 & 54 & 12 & 17.0 & 57 & 13 & 57 & 13 \\
\hline$I P P$ & 49 & 10 & 8.1 & 49 & 10 & 9.3 & 47 & 9 & 5.7 & 49 & 10 & 8.3 & 51 & 11 & 46 & 9 \\
\hline$S A V$ & 61 & 14 & 50.0 & 67 & 13 & 66.2 & 61 & 14 & 45.9 & 65 & 13 & 59.4 & 59 & 14 & 55 & 13 \\
\hline$S H Y$ & 53 & 11 & 21.9 & 53 & 11 & 22.9 & 50 & 10 & 14.9 & 53 & 10 & 21.0 & 55 & 11 & 53 & 11 \\
\hline$D S F$ & 62 & 16 & 42.3 & 68 & 17 & 57.6 & 59 & 15 & 37.1 & 64 & 17 & 49.0 & 60 & 16 & 58 & 15 \\
\hline PSY-5 & - & - & - & - & - & - & - & - & - & - & - & - & - & - & - & - \\
\hline$A G G R-r$ & 53 & 12 & 22.3 & 55 & 12 & 27.7 & 56 & 12 & 27.7 & 54 & 12 & 25.4 & 51 & 11 & 58 & 11 \\
\hline$P S Y C-r$ & 59 & 13 & 33.5 & 61 & 13 & 35.8 & 56 & 12 & 35.8 & 58 & 12 & 26.4 & 61 & 16 & 60 & 16 \\
\hline$D I S C-r$ & 61 & 11 & 35.0 & 55 & 10 & 19.5 & 56 & 10 & 19.5 & 54 & 10 & 16.0 & 56 & 11 & 64 & 11 \\
\hline$N E G E-r$ & 62 & 13 & 48.1 & 66 & 13 & 57.6 & 60 & 13 & 57.6 & 63 & 13 & 46.6 & 63 & 14 & 61 & 14 \\
\hline INTR-r & 62 & 14 & 44.2 & 68 & 14 & 60.6 & 61 & 15 & 60.6 & 53 & 15 & 53.0 & 62 & 15 & 55 & 14 \\
\hline
\end{tabular}

Comparison group descriptives are reproduced from MMPI-2-RF Technical by Yossef S. Ben-Porath and Auke Tellegen. Copyright $\odot 2008,2011$ by the Regents of the University of Minnesota. Reproduced by permission of the University of Minnesota Press. All rights reserved. "Minnesota Multiphasic Personality Inventory-2-RF®" and "MMPI-2-RF®" are trademarks owned by the Regents of the University of Minnesota

(b) higher rates of elevation occur on scales assessing common clinical concerns in a given setting, (c) in general, the high frequency of clinical elevation across scales is consistent with the high rate of mental and physical health needs for veterans, and (d) use of nongendered norms are supported although gender-specific comparison groups offer useful, incremental information.

Those seeking services for individual substance use treatment report historic Juvenile Conduct Problems as well as current Behavioral and Externalizing Dysfunction and Substance Abuse. Those who were assessed on a PCT are characterized by elevations consistent with diagnostic criteria of PTSD (American Psychiatric Association 2013), including intrusive symptoms (Criteria B), avoidant and numbering behaviors (Criteria C), and negatively internalized thoughts and emotions (Criteria D). This alignment with PTSD diagnostic criteria is consistent with 
Table 4 Cohen d effect sizes for the validity, Higher-order, and restructured clinical scales

\begin{tabular}{|c|c|c|c|c|c|c|}
\hline \multirow[t]{2}{*}{ Scale } & \multicolumn{6}{|c|}{ Compared Groups } \\
\hline & $\begin{array}{l}\text { SUD- } \\
\text { PCT }\end{array}$ & $\begin{array}{l}\text { SUD- } \\
\text { PolyTrauma }\end{array}$ & $\begin{array}{l}\text { SUD- } \\
\text { Medical }\end{array}$ & $\begin{array}{l}\text { PCT- } \\
\text { PolyTrauma }\end{array}$ & $\begin{array}{l}\text { PCT- } \\
\text { Medical }\end{array}$ & $\begin{array}{l}\text { PolyTrauma- } \\
\text { Medical }\end{array}$ \\
\hline Validity & - & - & - & - & - & - \\
\hline$V R I N-r$ & 0.20 & 0.20 & 0.20 & 0.00 & 0.00 & 0.00 \\
\hline TRIN-r & -0.14 & -0.14 & -0.14 & 0.00 & 0.00 & 0.00 \\
\hline$F-r$ & -0.25 & 0.10 & 0.05 & 0.35 & 0.30 & -0.05 \\
\hline$F p-r$ & -0.29 & 0.07 & 0.00 & 0.36 & 0.29 & -0.07 \\
\hline Fs & -0.45 & -0.21 & -0.21 & 0.26 & 0.25 & 0.00 \\
\hline$F B S-r$ & -0.64 & -0.50 & -0.41 & 0.14 & 0.21 & 0.00 \\
\hline$R B S$ & 0.59 & -0.47 & -0.35 & -1.06 & -0.94 & 0.12 \\
\hline$L-r$ & -0.29 & -0.38 & -0.38 & -0.10 & 0.00 & 0.10 \\
\hline$K-r$ & 0.33 & -0.11 & 0.00 & -0.42 & -0.32 & 0.10 \\
\hline $\mathrm{H}-\mathrm{O}$ & - & - & - & - & - & - \\
\hline EID & -0.24 & 0.31 & 0.00 & 0.56 & 0.24 & -0.31 \\
\hline$T H D$ & 0.08 & 0.32 & 0.24 & 0.40 & 0.32 & -0.08 \\
\hline$B X D$ & 0.43 & 0.61 & 0.61 & 0.18 & 0.18 & 0.00 \\
\hline $\mathrm{RC}$ & - & - & - & - & - & - \\
\hline$R C d$ & -0.24 & 0.24 & 0.08 & 0.50 & 0.33 & -0.17 \\
\hline$R C 1$ & -0.54 & -0.54 & -0.46 & 0.00 & 0.08 & 0.08 \\
\hline$R C 2$ & -0.33 & 0.13 & -0.19 & 0.47 & 0.13 & -0.32 \\
\hline$R C 3$ & -0.08 & 0.09 & 0.00 & 0.17 & 0.08 & -0.09 \\
\hline$R C 4$ & 0.50 & 0.78 & 0.78 & 0.26 & 0.26 & 0.00 \\
\hline$R C 6$ & 0.07 & 0.38 & 0.22 & 0.32 & 0.15 & -0.16 \\
\hline$R C 7$ & -0.31 & 0.23 & 0.00 & 0.54 & 0.31 & -0.23 \\
\hline$R C 8$ & -0.15 & 0.78 & 0.16 & 0.32 & 0.32 & 0.00 \\
\hline$R C 9$ & 0.00 & 0.00 & 0.10 & 0.00 & 0.10 & 0.10 \\
\hline
\end{tabular}

A positive value reflect a higher mean for the first group listed in the compared dyad higher scores on the Anxiety scale, which is associated with various manifestations of PTSD (Sellbom et al. 2012a). The Medical and Polytrauma groups both had higher Cognitive Complaints, Neurological Complaints, and a sense of Malaise which likely reflects some health concerns common in both of these treatment settings. Those in Polytrauma also endorse higher Social Avoidance and Disaffiliativeness, reflecting a similarity with those seeking PTSD treatment.

Variation in the frequency of clinically significant scale elevations across groups occurred in a similar pattern as the mean difference analyses, which also supported the distinct presenting concerns and treatment needs for veterans across different VA settings. Of note, clinically significant elevations were relatively common across settings, which likely reflects the high rate of complex and comorbid mental health needs of veterans, as well as the centrality of trauma-experiences to their difficulties. To some extent, these elevations may also be due to the frequency of compensation and pension evaluations, which may impact response style in spite of invalid responding detection methods (e.g., Ingram et al. 2019; Nelson et al. 2011; Ray 2017).

Several trends are evident when current study findings are contrasted to the comparison groups provided in the technical manual (i.e., psychiatric inpatient and residential substance use treatment). Anxiety, Social Avoidance, Disaffiliativeness, and Somatic Concerns are consistently higher in veterans from the more recent sample. These moderately-sized differences likely reflect the broader scope of the current groups (e.g., the inclusion of a medical group) as well as the increasing centrality of trauma in the psychological and physical health of veterans receiving VA services (Fulton et al. 2014; Possemato et al. 2010; Seal et al. 2009).

Reflecting that the MMPI-2-RF comparison groups target different VA populations, the psychiatric inpatient comparison group has substantially higher Suicidal/ 
Table 5 Cohen d effect sizes for Somatic, cognitive, internalizing, externalizing, interpersonal, and PSY-5 scales

\begin{tabular}{|c|c|c|c|c|c|c|}
\hline \multirow[t]{2}{*}{ Scale } & \multicolumn{6}{|c|}{ Compared Groups } \\
\hline & $\begin{array}{l}\text { SUD- } \\
\text { PCT }\end{array}$ & $\begin{array}{l}\text { SUD- } \\
\text { PolyTrauma }\end{array}$ & $\begin{array}{l}\text { SUD- } \\
\text { Medical }\end{array}$ & $\begin{array}{l}\text { PCT- } \\
\text { PolyTrauma }\end{array}$ & $\begin{array}{l}\text { PCT- } \\
\text { Medical }\end{array}$ & $\begin{array}{l}\text { PolyTrauma- } \\
\text { Medical }\end{array}$ \\
\hline Somatic/Cognitive & - & - & - & - & - & - \\
\hline$M L S$ & -0.50 & -0.15 & -0.32 & 0.33 & 0.17 & -0.16 \\
\hline$G I C$ & -0.52 & -0.19 & -0.37 & 0.31 & 0.12 & -0.18 \\
\hline$H P C$ & -0.42 & -0.67 & -0.33 & -0.25 & 0.08 & 0.33 \\
\hline NUC & -0.36 & -0.50 & -0.36 & -0.14 & 0.00 & 0.14 \\
\hline$C O G$ & -0.28 & -0.50 & -0.07 & -0.22 & 0.21 & 0.43 \\
\hline Internalizing & - & - & - & - & - & - \\
\hline SUI & 0.00 & 0.37 & 0.12 & 0.37 & 0.12 & -0.26 \\
\hline$H L P$ & -0.21 & 0.14 & -0.20 & 0.36 & 0.00 & -0.34 \\
\hline$S F D$ & 0.00 & 0.32 & 0.16 & 0.33 & 0.17 & -0.17 \\
\hline$N F C$ & 0.00 & 0.26 & 0.17 & 0.26 & 0.17 & 0.26 \\
\hline$S T W$ & -0.25 & 0.08 & 0.00 & 0.33 & 0.25 & -0.08 \\
\hline$A X Y$ & -0.48 & 0.06 & -0.06 & 0.55 & 0.42 & -0.12 \\
\hline$A N P$ & -0.42 & -0.16 & -0.24 & 0.24 & 0.16 & -0.08 \\
\hline$B R F$ & -0.15 & 0.16 & 0.00 & 0.32 & 0.15 & -0.16 \\
\hline$M S F$ & -0.11 & 0.16 & 0.00 & 0.37 & 0.11 & -0.25 \\
\hline Externalizing & - & - & - & - & - & - \\
\hline$J C P$ & 0.40 & 0.38 & 0.48 & 0.00 & 0.08 & 0.08 \\
\hline$S U B$ & 0.62 & 1.08 & 0.88 & 0.42 & 0.24 & -0.17 \\
\hline$A G G$ & -0.16 & 0.16 & 0.08 & 0.33 & 0.24 & -0.08 \\
\hline$A C T$ & 0.08 & 0.16 & 0.25 & 0.08 & 0.17 & 0.09 \\
\hline Interpersonal & - & - & - & - & - & - \\
\hline$F M L$ & 0.00 & 0.26 & 0.25 & 0.26 & 0.25 & 0.00 \\
\hline$I P P$ & 0.00 & 0.21 & 0.00 & 0.21 & 0.00 & -0.21 \\
\hline$S A V$ & -0.44 & 0.00 & -0.30 & 0.44 & 0.15 & -0.30 \\
\hline$S H Y$ & 0.00 & 0.29 & 0.00 & 0.29 & 0.00 & -0.30 \\
\hline$D S F$ & -0.36 & 0.19 & -0.12 & 0.56 & 0.24 & -0.31 \\
\hline PSY-5 & - & - & - & - & - & - \\
\hline$A G G R-r$ & -0.17 & -0.25 & -0.08 & -0.08 & 0.08 & 0.17 \\
\hline$P S Y C-r$ & -0.15 & 0.24 & 0.08 & 0.40 & 0.24 & -0.17 \\
\hline$D I S C-r$ & 0.57 & 0.48 & 0.67 & -0.10 & 0.10 & 0.20 \\
\hline$N E G E-r$ & -0.31 & 0.15 & -0.08 & 0.46 & 0.23 & -0.23 \\
\hline INTR-r & -0.43 & 0.07 & 0.62 & 0.48 & 1.03 & 0.53 \\
\hline
\end{tabular}

A positive value reflects a higher mean for the first group listed in the compared dyad
Death Ideation scores than the groups included in this study. This scale is reliably associated with risk of selfharm (Gottfried et al. 2014) that could lead to psychiatric hospitalization. Along the same lines, the psychiatric inpatient comparison group had a slightly more elevated Ideas of Persecution scale score than settings identified within this study. The residential substance use treatment comparison group had higher Substance Abuse and Disconstraint scores, consistent with issues expected among individuals with severe addictive behaviors (Bryant and McNulty 2017).
Evaluation of gender differences in the PCT and Medical groups offers both support for the MMPI-2RF's use of non-gendered norms as well as nuanced comparison groups which should be used during interpretations. In general, differences between genders on PCT follow a pattern of more internalizing symptoms for females and greater externalizing symptoms for males. Most scales do not differ at a substantial magnitude and, importantly, the scales central to the assessment of PTSD do not vary substantially between genders (e.g., AXY, RCd, SFD, ANP, and SAV; 
Table 6 PCT and medical comparison groups for the validity, Higher-order, and restructured clinical scales by gender

\begin{tabular}{|c|c|c|c|c|c|c|c|c|c|c|c|c|c|c|}
\hline \multirow[b]{3}{*}{ Scale } & \multicolumn{7}{|l|}{ PCT } & \multicolumn{7}{|c|}{ Medical } \\
\hline & \multicolumn{3}{|c|}{ Males $(n=1923)$} & \multicolumn{4}{|c|}{ Females $(n=364)$} & \multicolumn{3}{|c|}{ Males $(n=1968)$} & \multicolumn{4}{|c|}{ Females $(n=279)$} \\
\hline & $M$ & $S D$ & $\% \geq 65$ & $M$ & $S D$ & $\% \geq 65$ & $d$ & $M$ & $S D$ & $\% \geq 65$ & $M$ & $S D$ & $\% \geq 65$ & $d$ \\
\hline \multicolumn{15}{|l|}{ Validity } \\
\hline$V R I N-r$ & 52.7 & 9.6 & - & 53.3 & 9.4 & - & 0.07 & 53.0 & 9.9 & - & 51.8 & 9.5 & - & 0.12 \\
\hline$T R I N-r$ & 57.3 & 6.6 & - & 57.0 & 6.4 & - & 0.05 & 57.5 & 6.6 & - & 57.5 & 6.7 & - & 0.00 \\
\hline$F-r$ & 85.0 & 19.6 & - & 83.4 & 19.1 & - & 0.08 & 78.5 & 19.6 & - & 79.3 & 20.1 & - & 0.04 \\
\hline$F p-r$ & 66.3 & 13.4 & - & 65.9 & 13.8 & - & 0.03 & 63.0 & 13.9 & - & 64.0 & 14.2 & - & 0.00 \\
\hline Fs & 77.1 & 19.9 & - & 77.8 & 19.6 & - & 0.04 & 73.5 & 19.2 & - & 75.8 & 20.6 & - & 0.12 \\
\hline$F B S-r$ & 73.5 & 14.0 & - & 78.5 & 13.9 & - & 0.36 & 71.4 & 14.5 & - & 78.3 & 14.4 & - & 0.48 \\
\hline$R B S$ & 80.0 & 16.8 & - & 80.5 & 16.1 & - & 0.03 & 76.5 & 16.8 & - & 79.2 & 16.5 & - & 0.16 \\
\hline$L-r$ & 55.3 & 10.1 & - & 55.0 & 11.5 & - & 0.03 & 55.6 & 10.0 & - & 53.7 & 9.7 & - & 0.19 \\
\hline$K-r$ & 39.8 & 9.2 & - & 40.2 & 8.7 & - & 0.04 & 41.7 & 9.5 & - & 41.8 & 9.4 & - & 0.01 \\
\hline \multicolumn{15}{|l|}{$\mathrm{H}-\mathrm{O}$} \\
\hline$E I D$ & 68.9 & 12.3 & $60.6 \%$ & 71.6 & 11.9 & $75.0 \%$ & 0.22 & 65.4 & 12.9 & $55.1 \%$ & 70.4 & 12.8 & $69.8 \%$ & 0.39 \\
\hline$T H D$ & 61.8 & 13.5 & $36.8 \%$ & 61.0 & 12.1 & $32.4 \%$ & 0.06 & 57.8 & 12.0 & $24.2 \%$ & 57.4 & 11.7 & $20.5 \%$ & 0.03 \\
\hline$B X D$ & 57.6 & 11.2 & $29.1 \%$ & 51.4 & 9.6 & $9.9 \%$ & 0.59 & 54.7 & 10.5 & $19.0 \%$ & 49.3 & 9.5 & $8.3 \%$ & 0.54 \\
\hline \multicolumn{15}{|l|}{$\mathrm{RC}$} \\
\hline$R C d$ & 68.4 & 11.7 & $65.3 \%$ & 70.6 & 10.9 & $73.1 \%$ & 0.19 & 64.6 & 12.1 & $51.3 \%$ & 69.1 & 11.4 & $66.9 \%$ & 0.38 \\
\hline$R C 1$ & 71.8 & 13.0 & $71.9 \%$ & 74.7 & 14.1 & $73.1 \%$ & 0.21 & 71.2 & 12.5 & 70.3 & 74.9 & 13.3 & $75.9 \%$ & 0.30 \\
\hline$R C 2$ & 70.0 & 14.5 & $67.5 \%$ & 71.4 & 14.4 & $70.6 \%$ & 0.10 & 67.7 & 15.5 & $59.5 \%$ & 71.0 & 15.6 & $68.3 \%$ & 0.21 \\
\hline$R C 3$ & 58.4 & 11.6 & $35.5 \%$ & 58.3 & 11.9 & $34.3 \%$ & 0.01 & 57.4 & 11.6 & $32.0 \%$ & 55.7 & 11.5 & $25.5 \%$ & 0.15 \\
\hline$R C 4$ & 59.4 & 11.6 & $64.8 \%$ & 56.9 & 10.8 & $27.2 \%$ & 0.22 & 55.7 & 10.7 & $23.0 \%$ & 53.4 & 10.0 & $13.3 \%$ & 0.22 \\
\hline RC6 & 62.2 & 13.3 & $42.5 \%$ & 62.6 & 13.0 & $45.1 \%$ & 0.03 & 59.0 & 12.9 & $33.2 \%$ & 60.5 & 13.3 & $40.6 \%$ & 0.11 \\
\hline$R C 7$ & 64.7 & 12.6 & $54.7 \%$ & 67.3 & 12.8 & $62.1 \%$ & 0.20 & 61.2 & 12.9 & $44.9 \%$ & 64.5 & 12.8 & $56.1 \%$ & 0.05 \\
\hline$R C 8$ & 64.4 & 12.8 & $47.6 \%$ & 62.5 & 12.2 & $41.8 \%$ & 0.15 & 60.2 & 12.1 & $33.5 \%$ & 58.7 & 11.8 & $29.9 \%$ & 0.13 \\
\hline$R C 9$ & 53.3 & 10.3 & $14.5 \%$ & 48.4 & 8.9 & $6.0 \%$ & 0.51 & 51.9 & 10.1 & $11.7 \%$ & 48.2 & 9.7 & $6.1 \%$ & 0.37 \\
\hline
\end{tabular}

Sellbom et al. 2012a). The most pronounced differences emerged on the PSY-5 scales with males exhibiting personality traits associated with greater aggression with poorer behavioral constraint. The Medical group had differences between genders across most of the scales; however, these differences were small in effect for all somatic and cognitive scales. Otherwise, elevations followed a similar pattern as seen in the PCT group and elsewhere (e.g., males report more frequent externalizing behavior problems and females have more internalizing symptoms).

In sum, MMPI-2-RF scores reflect clinical needs that vary depending on their reason for receiving services, and this variability is consistent with the diversity of physical and mental health needs of veterans treated across VA service locations. Accordingly, the results of this study offer support and guidance for use of the MMPI-2-RF as a contextually sensitive measure of physical and mental health in veterans. The pattern and frequency of substantive scale elevations observed in this study also highlight the importance of using current and contextually-appropriate comparison groups when evaluating the MMPI-2-RF within a veteran population.

Results should be considered within the context of this study's limitations. First, this investigation does not include external criteria on which to evaluate the criterion validity of MMPI-2-RF substantive scale scores. Although research has consistently supported scales as measuring their intended content for veterans, the magnitude of these associations has not been examined as a function of treatment settings. Thus, further context-specific evaluation of extra-test criteria would be useful in understanding differences in scores across evaluation settings. Second, owing to limitations imposed by the VA database used, some important demographic information was not available within this study (i.e., age, ethnicity, and education level of respondents). Likewise, absent more specific referral information, it is not possible to determine the precise clinical issues 
Table 7 PCT and medical comparison groups for somatic, cognitive, internalizing, externalizing, interpersonal, and PSY-5 scales by gender

\begin{tabular}{|c|c|c|c|c|c|c|c|c|c|c|c|c|c|c|}
\hline \multirow[b]{3}{*}{ Scale } & \multicolumn{7}{|l|}{ PCT } & \multicolumn{7}{|c|}{ Medical } \\
\hline & \multicolumn{3}{|c|}{ Males $(n=1923)$} & \multicolumn{4}{|c|}{ Females $(n=364)$} & \multicolumn{3}{|c|}{ Males $(n=1968)$} & \multicolumn{4}{|c|}{ Females $(n=279)$} \\
\hline & $M$ & $S D$ & $\% \geq 65$ & $M$ & $S D$ & $\% \geq 65$ & $d$ & $M$ & $S D$ & $\% \geq 65$ & $M$ & $S D$ & $\% \geq 65$ & $d$ \\
\hline \multicolumn{15}{|c|}{ Somatic/Cognitive } \\
\hline$M L S$ & 73.5 & 11.4 & $76.6 \%$ & 74.5 & 10.8 & $79.7 \%$ & 0.09 & 71.9 & 12.1 & $70.5 \%$ & 74.3 & 12.3 & $75.2 \%$ & 0.20 \\
\hline$G I C$ & 66.0 & 16.3 & $46.7 \%$ & 71.7 & 16.0 & $58.0 \%$ & 0.35 & 64.8 & 16.6 & $42.5 \%$ & 69.6 & 17.4 & $52.5 \%$ & 0.28 \\
\hline$H P C$ & 64.8 & 12.1 & $54.8 \%$ & 68.2 & 12.5 & $64.6 \%$ & 0.28 & 63.8 & 11.4 & $50.4 \%$ & 69.5 & 12.3 & $70.5 \%$ & 0.48 \\
\hline$N U C$ & 70.6 & 14.3 & $71.3 \%$ & 69.7 & 14.7 & $67.6 \%$ & 0.17 & 70.2 & 13.6 & $72.7 \%$ & 69.5 & 13.9 & $68.3 \%$ & 0.05 \\
\hline$C O G$ & 72.3 & 14.0 & $68.1 \%$ & 72.9 & 13.4 & $69.0 \%$ & 0.04 & 69.2 & 14.5 & $58.2 \%$ & 71.0 & 13.7 & $60.8 \%$ & 0.13 \\
\hline \multicolumn{15}{|c|}{ Internalizing } \\
\hline$S U I$ & 59.1 & 17.4 & $45.8 \%$ & 58.6 & 17.6 & $43.7 \%$ & 0.03 & 56.5 & 16.3 & $38.7 \%$ & 58.9 & 17.2 & $45.7 \%$ & 0.14 \\
\hline$H L P$ & 61.1 & 14.3 & $38.6 \%$ & 60.6 & 14.9 & $37.4 \%$ & 0.03 & 60.7 & 14.8 & $38.1 \%$ & 62.5 & 15.1 & $42.8 \%$ & 0.12 \\
\hline$S F D$ & 60.9 & 12.2 & $50.9 \%$ & 64.6 & 12.0 & $63.2 \%$ & 0.31 & 58.6 & 12.2 & $41.6 \%$ & 63.1 & 11.9 & $57.9 \%$ & 0.37 \\
\hline$N F C$ & 58.6 & 11.8 & $28.4 \%$ & 63.0 & 12.3 & $42.9 \%$ & 0.37 & 57.0 & 11.5 & $22.9 \%$ & 61.0 & 12.0 & $38.1 \%$ & 0.34 \\
\hline$S T W$ & 61.3 & 11.9 & $51.2 \%$ & 62.4 & 11.6 & $55.5 \%$ & 0.09 & 58.8 & 12.0 & $41.5 \%$ & 63.3 & 11.8 & $58.3 \%$ & 0.38 \\
\hline$A X Y$ & 78.3 & 15.6 & $80.5 \%$ & 78.9 & 15.7 & $81.7 \%$ & 0.04 & 72.5 & 17.0 & $66.6 \%$ & 75.5 & 16.9 & $74.8 \%$ & 0.18 \\
\hline$A N P$ & 63.9 & 12.5 & $58.5 \%$ & 60.7 & 12.0 & $47.3 \%$ & 0.26 & 61.6 & 13.1 & $45.8 \%$ & 60.9 & 13.0 & $45.3 \%$ & 0.05 \\
\hline$B R F$ & 58.3 & 12.6 & $22.3 \%$ & 64.5 & 14.7 & $43.1 \%$ & 0.45 & 56.3 & 12.1 & $16.3 \%$ & 62.2 & 15.1 & $34.2 \%$ & 0.43 \\
\hline$M S F$ & 48.2 & 8.5 & $7.2 \%$ & 53.0 & 10.4 & $20.1 \%$ & 0.51 & 47.7 & 8.2 & $6.4 \%$ & 52.6 & 9.8 & $18.0 \%$ & 0.54 \\
\hline \multicolumn{15}{|c|}{ Externalizing } \\
\hline$J C P$ & 55.8 & 12.7 & $20.9 \%$ & 52.6 & 10.7 & $9.6 \%$ & 0.27 & 53.9 & 11.9 & $15.4 \%$ & 50.3 & 10.7 & $9.4 \%$ & 0.32 \\
\hline$S U B$ & 56.9 & 13.1 & $25.2 \%$ & 54.5 & 12.3 & $18.4 \%$ & 0.19 & 53.9 & 12.0 & $16.8 \%$ & 50.6 & 10.3 & $10.4 \%$ & 0.30 \\
\hline$A G G$ & 60.5 & 12.6 & $38.7 \%$ & 54.7 & 10.5 & $17.0 \%$ & 0.50 & 57.6 & 12.8 & $31.5 \%$ & 53.6 & 11.3 & $19.1 \%$ & 0.33 \\
\hline$A C T$ & 51.3 & 11.3 & $16.2 \%$ & 52.8 & 12.6 & $22.5 \%$ & 0.13 & 49.3 & 10.8 & $11.7 \%$ & 50.8 & 11.9 & $16.9 \%$ & 0.13 \\
\hline \multicolumn{15}{|c|}{ Interpersonal } \\
\hline$F M L$ & 55.9 & 11.6 & $71.1 \%$ & 61.1 & 12.6 & $37.4 \%$ & 0.43 & 53.1 & 11.0 & $14.8 \%$ & 58.9 & 13.2 & $29.9 \%$ & 0.48 \\
\hline$I P P$ & 47.8 & 10.0 & $8.0 \%$ & 52.3 & 11.4 & $15.9 \%$ & 0.42 & 48.2 & 9.7 & $7.5 \%$ & 52.5 & 11.7 & $15.5 \%$ & 0.40 \\
\hline$S A V$ & 66.9 & 12.5 & $67.3 \%$ & 64.8 & 13.3 & $60.7 \%$ & 0.16 & 65.4 & 12.9 & $62.9 \%$ & 64.8 & 13.3 & $59.7 \%$ & 0.46 \\
\hline$S H Y$ & 53.1 & 10.5 & $21.8 \%$ & 55.0 & 10.9 & $28.3 \%$ & 0.18 & 52.7 & 10.2 & $19.7 \%$ & 55.2 & 11.8 & $29.9 \%$ & 0.23 \\
\hline$D S F$ & 68.1 & 17.4 & $58.5 \%$ & 65.1 & 16.9 & $53.3 \%$ & 0.17 & 65.3 & 17.3 & $50.2 \%$ & 62.9 & 16.1 & $46.4 \%$ & 0.14 \\
\hline \multicolumn{15}{|l|}{ PSY-5 } \\
\hline$A G G R-r$ & 56.3 & 12.0 & $31.0 \%$ & 48.8 & 10.2 & $10.2 \%$ & 1.57 & 55.2 & 11.5 & $26.2 \%$ & 49.0 & 10.5 & $12.9 \%$ & 0.56 \\
\hline$P S Y C-r$ & 61.0 & 13.0 & $46.2 \%$ & 59.5 & 11.4 & $29.9 \%$ & 0.12 & 57.4 & 11.9 & $25.4 \%$ & 56.9 & 11.3 & $22.3 \%$ & 0.04 \\
\hline$D I S C-r$ & 56.3 & 10.3 & $22.3 \%$ & 49.5 & 8.3 & $4.4 \%$ & 0.73 & 54.4 & 9.5 & $15.7 \%$ & 47.4 & 8.3 & $4.0 \%$ & 0.78 \\
\hline$N E G E-r$ & 66.0 & 12.9 & $56.6 \%$ & 67.2 & 12.3 & $62.9 \%$ & 0.10 & 62.8 & 13.3 & $47.5 \%$ & 66.4 & 13.0 & $60.1 \%$ & 0.27 \\
\hline INTR-r & 68.5 & 14.0 & $61.0 \%$ & 67.6 & 14.5 & $58.2 \%$ & 0.06 & 67.2 & 14.6 & $55.5 \%$ & 68.2 & 14.7 & $56.1 \%$ & 0.07 \\
\hline
\end{tabular}

leading to the evaluation; however, stop codes do provide some information about likely clinical needs. Along the same lines, it was not possible to identify groups of veterans receiving inpatient psychiatric or substance abuse treatment to directly compare current MMPI-2-RF scores in these settings to the pre-existing MMPI-2-RF comparison groups.

Despite these limitations, this study supplements available information on use of, and research with, the MMPI-2-RF in veterans receiving care at the VA. This study documents a pattern of scale elevation for common presenting concerns that varies by treatment setting in expected ways. It also reports descriptive findings for these settings, which in turn provides contemporary comparison group data that can be used to guide veteran care in these settings.

\section{Compliance with Ethical Standards}

Conflict of Interest Paul B. Ingram, Anthony M. Tarescavage, Yossef S. Ben-Porath, and Mary E. Oehlert declare that they have no conflict of interest. 


\section{References}

Agha, Z., Lofgren, R. P., VanRuiswyk, J. V., \& Layde, P. M. (2000).

Are patients at Veterans Affairs medical centers sicker? A comparative analysis of health status and medical resource use. Archives of Internal Medicine, 160, 3252-3257. https://doi.org/10.1001/ archinte.160.21.3252.

American Psychiatric Association. (2013). Diagnostic and statistical manual of mental disorders (5th ed.). Washington, DC: Author.

Anestis, J. C., Finn, J. A., Gottfried, E., Arbisi, P. A., \& Joiner, T. E. (2015). The utility of the MMPI-2 restructured form validity scales in prediction of premature termination. Assessment, 22(3), 279-288. https://doi.org/10.1177/1073191114541672.

Arbisi, P. A., Sellbom, M., \& Ben Porath, Y. S. (2008). Empirical correlates of the MMPI -2 restructured clinical scales in psychiatric inpatients. Journal of Personality Assessment, 90, 122-128.

Arbisi, P. A., Rusch, L., Polusny, M. A., Thuras, P. E., \& Erbes, C. R. (2013). Does cynicism play a role in failure to obtain needed care? Mental health service utilization among returning U.S. National Guard soldiers. Psychological Assessment, 25(3), 991-996. https:// doi.org/10.1037/a0032225.

Bagalman, E. (2014). The Number of Veterans That Use VA Health Care Services: A Fact Sheet. Congressional research service. Retrieved from https://fas.org/sgp/crs/misc/R43579.pdf. Accessed 15 June 2018.

Ben-Porath, Y., \& Tellegen, A. (2008). Minnesota multiphasic personality inventory-restructured form: Manual for administration, scoring, and interpretation. Minneapolis: University of Minnesota Press.

Bryant, W. T., \& McNulty, J. L. (2017). Which domain of the PSY-5 is the most relevant to substance use? Journal of Personality Assessment, 99(5), 524-533. https://doi.org/10.1080/00223891.2016.1250213.

Cohen, B. E., Gima, K., Bertenthal, D., Kim, S., Marmar, C. R., \& Seal, K. H. (2010). Mental health diagnoses and utilization of VA nonmental health medical services among returning Iraq and Afghanistan veterans. Journal of General Internal Medicine, 25(1), 18-24

Cohen, J. (1988). Statistical Power Analysis for the Behavioral Sciences, 2nd Edition. Hillsdale, N.J.: Lawrence Erlbaum.

DeViva, J. C., \& Bloem, W. D. (2003). Symptom exaggeration and compensation seeking among combat veterans with posttraumatic stress disorder. Journal of Traumatic Stress, 16, 503-507. https://doi.org/ 10.1023/A:1025766713188.
Duggan, M., Rosenheck, R., \& Singleton, P. (2010). Federal policy and the rise in disability enrollment: Evidence for the veterans affairs' disability compensation program. Journal of Law and Economics, 53, 379-398. https://doi.org/10.1086/648385.

Forbey, J. D., Ben Porath, Y. S., \& Arbisi, P. A. (2012). The MMPI-2 computer adaptive version (MMPI-2 CA) in a medical outpatient facility. Psychological Assessment, 24, 628-639.

Fortney, J. C., Unützer, J., Wrenn, G., Pyne, J., Smith, G. R., Schoenbaum, M., \& Harbin, H. T. (2017). A Tipping Point for Measurement-Based Care. Psychiatric Services, 68, 179-188. https://doi.org/10.1176/appi.ps.201500439.

Freeman, T., Powell, M., \& Kimbrell, T. (2008). Measuring symptom exaggeration in veterans with chronic posttraumatic stress disorder. Psychiatry Research, 158, 374-380. https://doi.org/10.1016/j. psychres.2007.04.002.

Fulton, J. J., Calhoun, P. S., Wagner, H. R., Schry, A. R., Hair, L. P., Elbogen, E., \& Beckham, J. C. (2014). The prevalence of posttraumatic stress disorder in operation enduring freedom/ operation Iraqi freedom (OEF/OIF) veterans: A meta-analysis. Journal of Anxiety Disorders, 31, 98-107. https://doi.org/10. 1016/j.janxdis.2015.02.003.

Goodwin, B. E., Sellbom, M., \& Arbisi, P. A. (2013). Posttraumatic stress disorder in veterans: The utility of the MMPI-2-RF validity scales in detecting overreported symptoms. Psychological Assessment, 25, 671-678. https://doi.org/10.1037/a0032214.

Gottfried, E., Bodell, L., Carbonell, J., \& Joiner, T. (2014). The clinical utility of the MMPI-2-RF suicidal/death ideation scale. Psychological Assessment, 26, 1205-1211. https://doi.org/10.1037/ pas0000017.

Hankin, C. S., Spiro, A., Miller, D. R., \& Kazis, L. (1999). Mental disorders and mental health treatment among U.S. Department of Veterans Affairs outpatients: The veterans health study. Psychiatry, 156(12), 1924-1930.

Hoge, C. W., Auchterlonie, J. L., \& Miliken, C. S. (2006). Mental health problems, use of mental health services, and attrition from military service after returning from deployment to Iraq or Afghanistan. Journal of the American Medical Association, 295(9), 1023-1032.

Ingram, P. B., \& Ternes, M. (2016). The detection of content-based invalid responding: A meta-analysis of the MMPI-2-restructured Form's (MMPI-2-RF) over-reporting scales. The Clinical Neuropsychologist, 30, 473-496. https://doi.org/10.1080/ 13854046.2016.1187769.

Ingram, P. B., Tarescavage, A. M., Ben-Porath, Y. S., \& Oehlert, M. E. (2019). The MMPI-2-Restructured Form (MMPI-2-RF) Validity Scales: Patterns Observed across Veteran Affairs Settings [Measurement-Based Care and Psychological Assessment in Mental Health Services]. Psychological Services. https://doi.org/ $10.1037 / \mathrm{ser} 0000339$.

Kang, H. K., Bullman, T. A., Smolenski, D. J., Skopp, N. A., Gahm, G. A., \& Reger, M. A. (2015). Suicide risk among 1.3 million veterans who were on active duty during the Iraq and Afghanistan wars. Annals of Epidemiology, 25, 96-100. https://doi.org/10.1016/j. annepidem.2014.11.020.

Kaplan, M. S., Huguet, N., McFarland, B. H., \& Newsom, J. T. (2007). Suicide among male veterans: A prospective population-based study. Epidemiology and Community Health, 61(7), 619-624. https://doi.org/10.1136/jech.2006.054346.

Kazis, L. E., Miller, D. R., Clark, J., Skinner, K., Lee, A., Rogers, W., Spiro, A., Payne, S., Fincke, G., Selim, A., \& Linzer, M. (1998). Health-related quality of life in patients served by the Department of Veterans Affairs: Results from the veterans health study. Archives of Internal Medicine, 158, 626-632. https://doi.org/10.1001/archinte. 158.6.626.

Kilpatrick, D. G., Resnick, H. S., Milanak, M. E., Miller, M. W., Keyes, K. M., \& Friedman, M. J. (2013). National Estimates of Exposure to Traumatic Events and PTSD Prevalence Using DSM-IV and DSM-5 
Criteria. Journal of Traumatic Stress, 26, 537-547. https://doi.org/ $10.1002 /$ jts.21848.

Koffel, E., Polusny, M. A., Arbisi, P. A., \& Erbes, C. R. (2012). A preliminary investigation of the new and revised symptoms of posttraumatic stress disorder in DSM-5. Depression and Anxiety, 29(8), 731-738. https://doi.org/10.1002/da.21965.

National Center for Veteran Analysis and Statistics. (2016). U.S. veterans eligibility trends statistics [fact sheet]. Retrieved from https://www. va.gov/vetdata/. Accessed 15 June 2018.

Nelson, K. M., Starkebaum, G. A., \& Reiber, G. E. (2007). Veterans using and uninsured veterans not using Veteran Affairs (VA) health care. Public Health Reports, 122, 93-100. https://doi.org/10.1177/ 003335490712200113.

Nelson, N. W., Hoelzle, J. B., McGuire, K. A., Sim, A. H., Goldman, D. J., Ferrier-Auerbach, A. G., Charles, M. J., Arbisi, P. A., \& Sponheim, S. R. (2011). Self-report of psychological function among OEF/OIF personnel who also report combat-related concussion. The Clinical Neuropsychologist, 25, 716-740. https://doi.org/ 10.1080/13854046.2011.579174.

Nelson, N. W., Anderson, C. R., Hoelzle, J. B., \& Arbisi, P. A. (2014). Psychological assessment of veterans in outpatient mental health settings. In S. S. Bush (Ed.), Psychological assessment of veteran (pp. 17-50). Oxford: Oxford University Press.

Possemato, K., Wade, M., Andersen, J., \& Ouimette, P. (2010). The impact of PTSD, depression, and substance use disorders on disease burden and health care utilization among OEF/OIF veterans. Psychological Trauma: Theory, Research, Practice, and Policy, 2, 218-223. https://doi.org/10.1037/a0019236.

Ray, C. L. (2017). Practical use of MMPI-2-RF validity indicators in VA compensation and pension examination. Psychology Injury and Law, 10, 223-233. https://doi.org/10.1007/s12207-017-9289-3.

Russo, A. C. (2013). Ethical, legal, and risk management considerations in neuropsychological assessments of veterans. Psychology Injury and Law, 6, 21-30.

Russo, A. C. (2018). A practitioner survey of Department of Veterans Affairs Psychologists who provide neuropsychological assessments. Archives of Clinical Neuropsychology, 33, 1046-1059. https://doi. org/10.1093/arclin/acx139.

Seal, K. H., Bertenthal, D., Miner, C. R., Sen, S., \& Marmar, C. (2007). Bringing the war Back home- mental health disorders among 103788 US veterans returning from Iraq and Afghanistan seen at Department of Veterans Affairs Facilities. Archives of Internal Medicine, 167, 476-482. https://doi.org/10.1001/archinte.167.5.476.

Seal, K. H., Bertenthal, D., Maguen, S., Gima, K., Chu, A., \& Marmar, C. R. (2008). Getting beyond "Don't ask; Don't tell": An evaluation of US veterans administration Postdeployment mental health screening of veterans returning from Iraq and Afghanistan. American Journal of Public Health, 98, 714-720. https://doi.org/10.2105/AJPH.2007.115519.

Seal, K. H., Metzler, T. J., Gima, K., Bertenthal, D., Maguen, S., \& Marmar, C. R. (2009). Trends and risk factors for mental health diagnoses among Iraq and Afganistan veterans using Department of Veteran Affairs Health Care, 2002-2008. American Journal of Public Health, 99, 1651-1658. https://doi.org/10.2105/AJPH. 2008.150284.

Sellbom, M., Lee, T. T., Ben-Porath, Y. S., Arbisi, P. A., \& Gervais, R. O. (2012a). Differentiating PTSD symptomatology with the MMPI-2RF (restructured form) in a forensic disability sample. Psychiatry Research, 197, 172-179. https://doi.org/10.1016/j.psychres.2012. 02.003 .

Sellbom, M., Wygant, D. B., \& Bagby, R. M. (2012b). Utility of the MMPI-2-RF in detecting non-credible somatic complaints. Psychiatry Research, 197, 195-201.

Shekelle, P.G., Asch, S., Glassman, P., Matula, S., Trivedi, A., \& MiakeLye, I. (2010). Comparison of Quality of Care in VA and Non-VA Settings: A systematic Review. VA-ESP Project \# 05-226.

Shura, R. D., Denning, J. H., Miskey, H. M., \& Rowland, J. A. (2017). Symptom and performance validity with veterans assessed for attention-deficit/hyperactivity disorder (ADHD). Psychological Assessment, 29(12), 1458-1465. https://doi.org/10.1037/ pas0000436.

Tarescavage, A. M., Alosco, M., Ben-Porath, Y. B., Jones, L., \& Wood, A. (2015). Minnesota multiphasic personality Inventory-2-restructured form (MMPI-2-RF) scores generated from the MMPI-2 and MMPI-2-RF test booklets: Internal structure comparability in a sample of criminal defendants. Assessment, 22, 188-197.

Tellegen, A., \& Ben-Porath, Y. S. (2008/2011). The Minnesota multiphasic personality Inventory-2 restructured form: Technical manual. Minneapolis: University of Minnesota Press.

United States Department of Veterans Affair, National Center for Veterans Analysis and Statistics. (2017). VA Utilization Profile FY 2016 (Profile of Veterans: 2009) [Fact Sheet]. Retrieved from https://www.va.gov/vetdata/Quick_Facts.asp. Accessed 15 June 2018.

Wagner, T. H., Harris, K. M., Federman, B., Dai, L., Luna, Y., \& Humphreys, K. (2007). Prevalence of substance use disorders among veterans and comparable nonveterans from the National Survey on drug use and health. Psychological Services, 4, 149157. https://doi.org/10.1037/1541-1559.4.3.149.

Wilson, N. J., \& Kizer, K. W. (1997). The VA health care system: An unrecognized national safety net. Health Affairs, 16(4), 200-204.

Wolf, E. J., Miller, M. W., Orazem, R. J., Weierich, M. R., Castillo, D. T., Milford, J., Kaloupek, D. G., \& Keane, T. M. (2008). The MMPI-2 Restructured Clinical Scales in the assessment of post-traumatic stress disorder with comorbid disorders. Psychological Assessment, 20, 327-340. https://doi.org/10.1037/a0012948.

Worthen, M. D., \& Moering, R. D. (2011). A practical guide to conducting VA compensation and pension exams for PTSD and other mental disorders. Psychological Injury and Law, 4, 187-216. https://doi.org/10.1007/s12207-011-9115-2. 